Pacific Journal of Mathematics

OINT BasIC SUBGRoups 


\section{DISJOINT BASIC SUBGROUPS}

\section{A. Richard Mitchell and Roger W. Mitchell}

This paper arose from consideration of the following questions. First, what characterizes those infinite Abelian reduced $p$-groups which possess disjoint basic subgroups? Second, are there properties that a basic subgroup must possess to insure the existence of a basic subgroup disjoint from it?

We show that a necessary and sufficient condition for an infinite Abelian reduced $p$-group $G$ to contain disjoint basic subgroups is that $|G|=$ final rank $G$. Futhermore, in such a group a necessary and sufficient condition for a basic subgroup $B$ to have a basic subgroup disjoint from it is that $B$ is a lower basic subgroup of $G$.

Throughout this paper the word "group" will mean "Abelian group" and the notation used will be that of L. Fuchs in [1] with the exception that $A \oplus B$ will denote the direct sum of the groups $A$ and $B$, and $A+B$ will be the, not necessarily direct, sum.

We will use the following theorem:

Theorem A. (Mitchell and Mitchell in [4]) Let G be an infinite reduced Abelian p-group and $B$ a basic subgroup of $G$ such that $G / B=$ $\sum_{\alpha} \in_{I}\left(G_{\alpha} / B\right)$ where $G_{\alpha} / B \cong Z\left(p^{\infty}\right)$ for all $\alpha \in I$. Then $G=H \oplus K$ and $B=H \oplus L$ where $L$ is a basic subgroup of $K$ such that $r(K / L)=$ $r(G / B)=|I|$ and $|K|=\operatorname{maximum}\left\{\boldsymbol{\aleph}_{0},|I|\right\}$.

We first prove the following lemmas:

LEMma 1. Let $G$ be a p-group without elements of infinite height, and such that final rank $(G)=|G|$. Let $B$ be a lower basic subgroup of $G$. Then there exists a basic subgroup, $B^{\prime}$, of $G$ which is disjoint from $B$.

Proof. Let $B=\sum_{\alpha \in_{I}}\left\langle y_{\alpha}\right\rangle$, and let $G / B=\sum_{\beta \in J} C_{\beta}$ where each $C_{\beta} \cong Z\left(p^{\infty}\right)$. Let $\left\{\left\{y_{\alpha} \mid \alpha \in I\right\},\left\{c_{\beta, n} \mid \beta \in J, n=1,2, \cdots\right\}\right\}$ be a quasibasis for $G$. Since $B$ is a lower basic subgroup of $G$ and final rank $(G)=|G|$, we have $|J|=r(G / B)=|G| \geqq|B| \geqq|I|$. If indeed we have $|J|>|I|$ a pure subgroup $H$ of $G$ can be chosen such that $H \supset B, H^{1}=0$, and final $\operatorname{rank}(H)=|H|=|I|$. We can then prove that there is a basic subgroup $B^{\prime}$ of $H$ which is disjoint from $B$ and $H$ being pure in $G$ will insure $B^{\prime}$ is a basic subgroup of $H$. Thus it suffices to complete the proof when $|J|=|I|$ and we will assume moreover $I=J$. 
Now for each $\alpha \in I$ choose from $\left\{c_{\alpha, n}\right\}_{n=1}^{\infty}$ the element $c_{\alpha, 2 E\left(y_{\alpha}\right)}$. Define $B^{\prime}=\left\langle\left\{y_{\alpha}-p^{E\left(y_{\alpha}\right)} c_{\alpha, 2 E\left(y_{\alpha}\right)}\right\}_{\alpha} \in_{I}\right\rangle$. We now claim that $B^{\prime}$ is the desired basic subgroup of $G$ which is disjoint from $B$. To see this we prove the following:

(i ) First claim that

$$
B^{\prime}=\sum_{\alpha \in I}\left\langle y_{\alpha}-p^{E\left(y_{\alpha}\right)} c_{\alpha, 2 E\left(y_{\alpha}\right)}\right\rangle .
$$

Suppose that

$$
0=\sum_{i=1}^{n} a_{i}\left(y_{\alpha_{i}}-p^{E\left(y_{\alpha_{i}}\right)} c_{\alpha_{i}, 2 E\left(y_{\alpha_{i}}\right)}\right),
$$

then

$$
\sum_{i=1}^{n} a_{i} y_{\alpha_{i}}=\sum_{i=1}^{n} a_{i} p^{E\left(y_{\left.\alpha_{i}\right)}\right.} c_{\alpha_{i}, 2 E\left(y_{\alpha_{i}}\right)}
$$

Since the

$$
E\left(y_{\alpha}-p^{E\left(y_{\alpha}\right)} c_{\alpha, 2 E\left(y_{\alpha}\right)}\right)=E\left(y_{\alpha}\right),
$$

we would be finished if $\sum_{i=1}^{n} a_{i} y_{\alpha_{i}}=0$, so we can assume that $\sum_{i=1}^{n} a_{i} y_{\alpha_{i}} \neq 0$, and without loss of generality $a_{i}$ is not equal to 0 $\bmod o\left(y_{\alpha_{i}}\right)$. Now the height $h_{\theta}\left(\sum_{i=1}^{n} a_{i} y_{\alpha_{i}}\right)=r$, where $r$ is the largest positive integer such that $p^{r}$ divides each $a_{i}$, since $\sum_{i=1}^{n} a_{i} y_{\alpha_{i}}$ is an element of $B=\sum_{\alpha \epsilon_{I}}\left\langle y_{\alpha}\right\rangle$. But,

$$
h_{G}\left(\sum_{i=1}^{n} a_{i} p^{E\left(y_{\alpha_{i}}\right)} c_{\alpha_{i}, 2 E\left(y_{\alpha_{i}}\right)}\right) \geqq \underset{i=1,2, \cdots, n}{\operatorname{minimum}}\left\{r+E\left(y_{\alpha_{i}}\right)\right\}>r,
$$

which contradicts the equality

$$
\sum_{i=1}^{n} a_{i} y_{\alpha_{i}}=\sum_{i=1}^{n} a_{i} p^{\mathbb{E}\left(y_{\left.\alpha_{i}\right)}\right)} c_{\alpha_{i}, 2 E\left(y_{\alpha_{i}}\right)} .
$$

Therefore, we must have that

$$
B^{\prime}=\sum_{\alpha \in I}\left\langle y_{\alpha}-p^{E\left(y_{\alpha}\right)} c_{\alpha, 2 E\left(y_{\alpha}\right)}\right\rangle .
$$

(ii) Next we will show that $B^{\prime}$ is a pure subgroup of $G$. Let $z \in B^{\prime}[p]$, and write

$$
z=\sum_{i=1}^{n} a_{i} p^{E\left(y_{\alpha_{i}}\right)-1}\left(y_{\alpha_{i}}-p^{E\left(y_{\alpha_{i}}\right)} c_{\alpha_{i}, 2 E\left(y_{\alpha_{i}}\right)}\right)
$$

where each $a_{i}$ is relatively prime to $p$. Now we have that

$$
\begin{aligned}
h_{B^{\prime}}(z) & =\underset{i=1, \cdots, n}{\operatorname{minimum}}\left\{h_{B^{\prime}}\left[a_{i} p^{E\left(y_{\alpha_{i}}\right)-1}\left(y_{\alpha_{i}}-p^{E\left(y_{\alpha_{i}}\right)} c_{\alpha_{i}, 2 E\left(y_{\alpha_{i}}\right)}\right)\right]\right\} \\
& =\operatorname{minimum}_{i=1, \cdots, n}\left\{E\left(y_{\alpha_{i}}\right)-1\right\} .
\end{aligned}
$$


But

$$
h_{G}(z)=h_{G}\left[\left(\sum_{i=1}^{n} a_{i} p^{E\left(y_{\alpha_{i}}\right)-1} y_{\alpha_{i}}\right)-\left(\sum_{i=1}^{n} a_{i} p^{2 E\left(y_{\alpha_{i}}\right)-1} c_{\alpha_{i}, 2 E\left(y_{\alpha_{i}}\right)}\right)\right],
$$

and

$$
\begin{aligned}
h_{G}\left(\sum_{i=1}^{n} a_{i} p^{2 E\left(y_{\alpha_{i}}\right)-1} c_{\alpha_{i}, 2 E\left(y_{\alpha_{i}}\right)}\right) & \geqq \operatorname{minimum}\left\{2 E\left(y_{\alpha_{i}}\right)-1\right\} \\
& >\operatorname{minimum}_{i=1, \cdots, n}\left\{E\left(y_{\alpha_{i}}\right)-1\right\},
\end{aligned}
$$

and

$$
h_{G}\left(\sum_{i=1}^{n} a_{i} p^{E\left(y_{\alpha_{i}}\right)-1} y_{\alpha_{i}}\right)=\operatorname{minimum}_{i=1, \cdots, n}\left\{E\left(y_{\alpha_{i}}\right)-1\right\} .
$$

Since the height of the sum of two elements with different heights is just the height of the smaller, we have that

$$
h_{\theta}(z)=\underset{i=1, \cdots, n}{\operatorname{minimum}}\left\{E\left(y_{\alpha_{i}}\right)-1\right\} \text {. }
$$

Therefore $h_{G}(z)=h_{B^{\prime}}(z)$ for each element $z \in B^{\prime}[p]$, and hence by Lemma 7, page 20, in [3], we have that $B^{\prime}$ is pure.

(iii) We will now complete the proof that $B^{\prime}$ is a basic subgroup of $G$ by showing that $B^{\prime}$ cannot be extended to a larger pure direct sum of cyclic groups. Suppose that $B^{\prime} \oplus\langle z\rangle$ is a pure direct sum of cyclic groups. Since $\left\{\left\{y_{\alpha} \mid \alpha \in I\right\},\left\{c_{\alpha, n} \mid \alpha \in I, n=1,2, \cdots\right\}\right\}$ is a quasibasis for $G$, we can write

$$
z=\sum_{i=1}^{n} a_{i} y_{\alpha_{i}}+\sum_{i=1}^{k} s_{j} c_{\alpha_{j}, r_{j}} .
$$

Now we also know that $c_{\alpha_{j}, r_{j}}=p c_{\alpha_{j}, r_{j}+1}+b_{j}$ where $b_{j} \in B=\sum_{\alpha \epsilon_{I}}\left\langle y_{\alpha}\right\rangle$, hence we can write

$$
z=\sum_{j=1}^{k} s_{j} p c_{\alpha_{j}, r_{j}+1}+\sum_{i=1}^{m} t_{i} y_{\alpha_{i}} .
$$

Now write

$$
z=\sum_{j=1}^{k} s_{j} p c_{\alpha_{j}, r_{j}+1}+\sum_{i=1}^{m_{1}} t_{i}^{\prime} y_{\alpha_{i}}+\sum_{i=1}^{m_{2}} t_{i}^{\prime \prime} y_{\alpha_{i}}
$$

where each $t_{i}^{\prime}$ is divisible by $p$, and each $t_{i}^{\prime \prime}$, is relatively prime to $p$. We are assuming that $H=B^{\prime} \oplus\langle z\rangle$ is pure in $G$, and hence, $h_{\theta}(z)=$ $h_{H}(z)=0$, and since $H$ is a direct sum of cyclic groups we must also have that $h_{H}\left(b^{\prime}+z\right)=0$, for any $b^{\prime} \in B$. Consider the following element of $B^{\prime}$,

$$
\sum_{i=1}^{m_{2}} t_{i}^{\prime \prime}\left(y_{\alpha_{i}}-p^{E\left(y_{\left.\alpha_{i}\right)} c_{\alpha_{i}, 2 E\left(y_{\alpha_{i}}\right)}\right)}\right.
$$


Now we have

$$
\begin{aligned}
z- & \sum_{i=1}^{m_{2}} t_{i}^{\prime \prime}\left(y_{\alpha_{i}}-p^{E\left(y_{\alpha_{i}}\right)} c_{\alpha_{i}, 2 E\left(y_{\alpha_{i}}\right)}\right) \\
& =\sum_{j=1}^{k} s_{j} p c_{\alpha_{j}, r_{j}+1}+\sum_{i=1}^{m_{1}} t_{i}^{\prime} y_{\alpha_{i}}+\sum_{i=1}^{m_{2}} t_{i}^{\prime \prime} p^{E\left(y_{\alpha_{i}}\right)} c_{\alpha_{i}, 2 E\left(y_{\alpha_{i}}\right)},
\end{aligned}
$$

thus

$$
h_{G}\left(z-\sum_{i=1}^{m_{2}} t_{i}^{\prime \prime}\left(y_{\alpha_{i}}-p^{E\left(y_{\left.\alpha_{i}\right)}\right.} c_{\alpha_{i}, 2 E\left(y_{\left.\alpha_{i}\right)}\right)}\right) \geqq 1,\right.
$$

but this contradicts the assumption that $H$ is a pure subgroup of $G$. Thus $B^{\prime}$ is a basic subgroup of $G$.

To complete the proof of the theorem, we need only show that $B \cap B^{\prime}=0$. To see this suppose that

$$
\sum_{j=1}^{k} s_{j}\left(y_{\alpha_{j}}-p^{E\left(y_{\left.\alpha_{j}\right)}\right.} c_{\alpha_{j}, 2 E\left(y_{\alpha_{j}}\right)}\right)=\sum_{i=1}^{n} a_{i} y_{\alpha_{i}} .
$$

Consider

$$
\begin{aligned}
\sum_{i=1}^{n} a_{i} y_{\alpha_{i}}+B=0+B & =\sum_{j=1}^{k} s_{j}\left(y_{\alpha_{j}}-p^{\mathbb{E}\left(y_{\alpha_{j}}\right)} c_{\alpha_{j}, 2 E\left(y_{\alpha_{j}}\right)}\right)+B \\
& =\sum_{j=1}^{k} s_{j} p^{E\left(y_{\left.\alpha_{j}\right)}\right)} c_{\alpha_{j}, 2 E\left(y_{\alpha_{j}}\right)}+B \\
& =\sum_{j=1}^{k} s_{j} c_{\alpha_{j}, \mathbb{E}\left(y_{\alpha_{j}}\right)}+B,
\end{aligned}
$$

so that $s_{j} c_{\alpha_{j}, E\left(y_{\left.\alpha_{j}\right)}\right.}+B=0+B$ for $j=1, \cdots, k$ since each is from a different summand of $G / B$. But this means that $s_{j}$ is divisible by $p^{E\left(y_{\alpha_{j}}\right)}$ for $j=1, \cdots, k$. Thus

$$
\sum_{j=1}^{k} s_{j}\left(y_{\alpha_{j}}-p^{E\left(y_{\alpha_{j}}\right)} c_{\alpha_{j}, 2 E\left(y_{\alpha_{i}}\right)}\right)=0,
$$

and so $B \cap B^{\prime}=0$.

LEMMA 2. Let $G$ be a reduced p-group such that final rank $(G)=|G|$. Let $B$ be a lower basic subgroup of $G$. Then there exists a basic subgroup $B^{\prime}$ of $G$ which is disjoint from $B$.

Proof. Let $H$ be a high subgroup of $G$ which contains $B$. By Theorem 5 in [2] $H$ is pure and a basic subgroup of $H$ is a basic of $G$. Thus $\operatorname{rank}(G / B)=\operatorname{rank}(H / B)+\operatorname{rank}(G / H)$, and we consider the following cases:

Case (i). Suppose that $\operatorname{rank}(H / B)=\operatorname{rank}(G / B)$, then we know that final $\operatorname{rank}(H) \geqq \operatorname{rank}(H / B)=\operatorname{rank}(G / B)=\operatorname{final} \operatorname{rank}(G)=|G| \geqq|H|$. Thus final rank $(H)=|H|$, and Lemma 1 completes the proof. 
Case (ii). Suppose that $\operatorname{rank}(G / B)>\operatorname{rank}(H / B)$. Since $|G|=$ final $\operatorname{rank}(G)$, and final $\operatorname{rank}(G)=\operatorname{rank}(G / B)$, we know that $\operatorname{rank}(G / B)$ is infinite. But if $\operatorname{rank}(G / B)>\operatorname{rank}(H / B)$, and is infinite, then the $\left|G^{1}[p]\right|$ is infinite, and hence we have $\left|G^{1}[p]\right|=\operatorname{rank}(G / H)>\operatorname{rank}(H / B)$. Now $\operatorname{rank}(G / B)=\operatorname{rank}(H / B)+\operatorname{rank}(G / H)=\operatorname{rank}(H / B)+\left|G^{1}[p]\right|$, and thus $\left|G^{1}[p]\right|=\operatorname{rank}(G / B)=|G|$. So that $\left|G^{1}[p]\right| \geqq|B|$, and for the purposes of this proof we can assume that $\left|G^{1}[p]\right|=|B|$. Let $G^{1}[p]=\sum_{\alpha \epsilon_{I}}\left\langle y_{\alpha}\right\rangle$, and let $B=\sum_{\alpha \epsilon_{I}}\left\langle x_{\alpha}\right\rangle$. For each $\alpha \in I$ choose $z_{\alpha}$ such that $y_{\alpha}=p^{E\left(x_{\alpha}\right)-1} z_{\alpha}$, which can be done since each $y_{\alpha}$ has infinite height. Now consider the subgroup $B^{\prime}=\left\langle\left\{x_{\alpha}+z_{\alpha}\right\}_{\alpha \in I}\right\rangle$. We claim that $B^{\prime}$ is a basic subgroup of $G$ which is disjoint from $B$. To see this we prove:

(i) First we must show that $B^{\prime}=\sum_{\alpha \epsilon_{I}}\left\langle x_{\alpha}+z_{\alpha}\right\rangle$. Suppose

$$
\sum_{i=1}^{n} a_{i}\left(x_{\alpha_{i}}+z_{\alpha_{i}}\right)=0,
$$

where $a_{i} \not \equiv 0 \bmod \left(o\left(x_{\alpha_{i}}\right)\right)$, and $a_{i}<o\left(x_{\alpha_{i}}\right)$. Notice that $o\left(x_{\alpha}\right)=o\left(x_{\alpha}+z_{\alpha}\right)$ since $p\left(y_{\alpha}\right)=0=p^{E\left(x_{\alpha}\right)} z_{\alpha}$. Let $k_{i}$ be the largest positive integer such that $p^{k_{i}}$ divides $a_{i}$. Let $r=$ maximum $_{i=1, \ldots, n}\left\{E\left(x_{\alpha_{i}}\right)-k_{i}\right\}$, and consider

$$
\begin{aligned}
0=p^{r-1}\left(\sum_{i=1}^{n} a_{i}\left(x_{\alpha_{i}}+z_{\alpha_{i}}\right)\right) & =\sum_{i=1}^{n} a_{i} p^{r-1} x_{\alpha_{i}}+\sum_{i=1}^{n} a_{i} p^{r-1} z_{\alpha_{i}} \\
& =\sum_{i=1}^{n} a_{i} p^{r-1} x_{\alpha_{i}}+\sum_{i=1}^{n} a_{i}^{\prime} y_{\alpha_{i}} .
\end{aligned}
$$

Hence we have

$$
\sum_{i=1}^{n} a_{i}^{\prime} y_{\alpha_{i}}=-\sum_{i=1}^{n} a_{i} p^{r-1} x_{\alpha_{i}},
$$

but this means that an element of infinite height is equal to an element of finite height which is contradiction. Thus $B^{\prime}=\sum_{\alpha \in I}\left\langle x_{\alpha}+z_{\alpha}\right\rangle$.

(ii) We must show that $B^{\prime}$ is pure. Let $s \in B^{\prime}[p]$, and write

$$
s=\sum_{i=1}^{n} a_{i} p^{E\left(x_{\alpha_{i}}\right)-1}\left(x_{\alpha_{i}}+z_{\alpha_{i}}\right)
$$

where $a_{i}$ is relatively prime to $p$ for each $i$. Since $B^{\prime}=\sum_{\alpha \in I}\left\langle x_{\alpha}+z_{\alpha}\right\rangle$, we know that $h_{B^{\prime}}(s)=$ minimum $_{i=1, \ldots, n}\left\{E\left(x_{\alpha_{i}}\right)-1\right\}$. Now consider

$$
\begin{aligned}
h_{G}(s) & =h_{\theta}\left(\sum_{i=1}^{n} a_{i} p^{E\left(x_{\alpha_{i}}\right)-1} x_{\alpha_{i}}+\sum_{i=1}^{n} a_{i} p^{E\left(x_{\alpha_{i}}\right)-1} z_{\alpha_{i}}\right) \\
& =h_{\theta}\left(\sum_{i=1}^{n} a_{i} p^{E\left(x_{\alpha_{i}}\right)-1} x_{\alpha_{i}}+\sum_{i=1}^{n} a_{i} y_{\alpha_{i}}\right) \\
& =h_{\theta}\left(\sum_{i=1}^{n} a_{i} p^{E\left(x_{\alpha_{i}}\right)-1} x_{\alpha_{i}}\right) \\
& =\operatorname{minimum}_{i=1, \cdots, n}\left\{E\left(x_{\alpha_{i}}\right)-1\right\} .
\end{aligned}
$$


Thus $B^{\prime}$ is a pure subgroup of $G$.

(iii) To complete the proof that $B^{\prime}$ is a basic subgroup of $G$, we need only prove that the quotient $G / B^{\prime}$ is divisible. If every element $s+B^{\prime} \in\left(G / B^{\prime}\right)[p]$ has infinite height then $G / B^{\prime}$ is divisible. Thus we can assume $h_{\theta \mid B^{\prime}}\left(s+B^{\prime}\right)=n$, a finite integer, and we can assume that $o(s)=o\left(s+B^{\prime}\right)$. Now since $G / B$ is divisible we know that $s+B$ has infinite height in $G / B$. Consider the following cases:

Case (a). Suppose that $s \in B$, then

$$
s=\sum_{i=1}^{m} a_{i} p^{E\left(x_{\alpha_{i}}\right)-1} x_{\alpha_{i}}
$$

where $a_{i}$ is relatively prime to $p$ for each $i$. Now define the following element of $B^{\prime}$, let

$$
b^{\prime}=\sum_{i=1}^{m} a_{i} p^{E\left(x_{\alpha_{i}}\right)-1}\left(x_{\alpha_{i}}+z_{\alpha_{i}}\right) .
$$

But

$$
s-b^{\prime}=\sum_{i=1}^{m} a_{i} y_{\alpha_{i}}, \quad \text { and } \quad \sum_{i=1}^{m} a_{i} y_{\alpha_{i}}
$$

has infinite height in $G$ so that $h_{G / B^{\prime}}\left(s+B^{\prime}\right)$ is infinite. Therefore $G / B^{\prime}$ must be divisible.

Case (b). Suppose that $s \notin B$, then there exists an element $\sum_{i=1}^{m} a_{i} x_{\alpha_{i}} \in B$, such that

$$
s+\sum_{i=1}^{m} a_{i} x_{\alpha_{i}}=p^{n+1} g
$$

since $h_{\theta \mid B}(s+B)$ is infinite. Now write

$$
\sum_{i=1}^{m} a_{i} x_{\alpha_{i}}=\sum_{j=1}^{r} c_{j} x_{\alpha_{j}}+\sum_{k=1}^{t} d_{k} x_{\alpha_{k}},
$$

where $c_{j}$ is divisible by $p^{\mathbb{E}\left(x_{\alpha_{j}}\right)-1}$, and $d_{k}$ is not divisible by $p^{\mathbb{E}\left(x_{\alpha_{k}}\right)^{1-1}}$. Thus

$$
s+\sum_{j=1}^{r} c_{j} x_{\alpha_{j}}+\sum_{k=1}^{t} d_{k} x_{\alpha_{k}}=p^{n+1} g
$$

and so multiplication by $p$ yields

$$
\sum_{k=1}^{t} p d_{k} x_{\alpha_{k}}=p^{n+2} g \text {. }
$$

By choice of the $x_{\alpha_{k}}$ 's we know $p d_{k} x_{\alpha_{k}} \neq 0$. Thus we must have

$$
h_{\theta}\left(\sum_{k=1}^{t} d_{k} x_{\alpha_{k}}\right) \geqq n+1 \text {. }
$$


Therefore by letting $c_{j}^{\prime}=c_{j} / p^{E\left(x_{\alpha_{j}}\right)-1}$ we have that

$$
s+\sum_{j=1}^{r} p^{E\left(x_{\alpha_{j}}\right)-1} c_{j}^{\prime} x_{\alpha_{j}}=p^{n+1} g^{\prime} .
$$

Consider the element $b^{\prime} \in B^{\prime}$ such that

$$
b^{\prime}=\sum_{j=1}^{r} c_{j}^{\prime} p^{E\left(x_{\alpha_{j}}\right)-1}\left(x_{\alpha_{j}}+z_{\alpha_{j}}\right)=\sum_{j=1}^{r} c_{j}^{\prime} p^{E\left(x_{\alpha_{j}}\right)-1} x_{\alpha_{j}}+\sum_{j=1}^{r} c_{j}^{\prime} y_{\alpha_{j}} .
$$

Then

$$
s-b^{\prime}=p^{n+1} g^{\prime}-\sum_{j=1}^{r} c_{j}^{\prime} y_{\alpha_{j}}=p^{n+1} g^{\prime \prime}
$$

since $\sum_{j=1}^{r} c_{j}^{\prime} y_{\alpha_{j}}$ has infinite height in $G$. But this implies that

$$
h_{\theta / B^{\prime}}\left(s+B^{\prime}\right) \geqq n+1
$$

which contradicts the assumption that $h_{a / B^{\prime}}\left(s+B^{\prime}\right)=n$. Thus $G / B^{\prime}$ must be divisible.

(iv) To complete the proof of the theorem, we need only show that $B \cap B^{\prime}=0$. To see this, suppose that

$$
\sum_{i=1}^{n} a_{i} x_{\alpha_{i}}=\sum_{j=1}^{k} s_{j}\left(x_{\alpha_{j}}+z_{\alpha_{j}}\right) \neq 0
$$

so

$$
\sum_{i=1}^{n} a_{i} x_{\alpha_{i}}-\sum_{j=1}^{k} s_{j} x_{\alpha_{j}}=\sum_{j=1}^{k} s_{j} z_{\alpha_{j}} \neq 0,
$$

and by multiplying both sides of this equation by an appropriate power of $p$ we get an element of infinite height on one side and an element of finite height on the other side, which is a contradiction. Thus $B \cap B^{\prime}=0$, and the proof is finished.

The following theorem gives a sufficient condition for a group $G$ to possess disjoint basic subgroups.

THEOREM 3. Let $G$ be a reduced Abelian p-group. If final rank $(G)=|G|$, then $G$ contains two disjoint basic subgroups.

Proof. Since every $p$-group has a lower basic subgroup, then Lemma 2 will complete the proof.

The next corollary shows that the restriction final $\operatorname{rank}(G)=|G|$, can be removed if instead of disjoint basic subgroups, one is seeking two basic subgroups whose intersection is bounded.

CoRollary 4. Let $G$ be a reduced Abelian p-group. Then there exists two basic subgroups of $G$ whose intersection is bounded. 
Proof. By Theorem 31.5, page 106, in [1], we can write $G=$ $H \oplus K$, where $K$ is bounded direct sum of cyclic groups, and final rank $(H)=|H|$. Now by Theorem 3 there exists $A$ and $B$ which are disjoint basic subgroups of $H$. Now $A \oplus K$ and $B \oplus K$ are basic subgroups of $G$ whose intersection is bounded.

TheOREM 5. Let $G$ be a reduced Abelian p-group, and suppose that $A$ and $B$ are two disjoint basic subgroups of $G$. Then rank $(G / A)=\operatorname{rank}(G / B)=|G|$.

Proof. Suppose that rank $(G / A)<|G|$, then by Lagrange's Theorem and since basic subgroups are isomorphic we know that $|G|=|B|=|A|$. By Theorem A we have $G=L \oplus F$ and $A=$ $A^{\prime} \oplus F$, where $|L|=\operatorname{maximum}\left\{\boldsymbol{S}_{0}, \operatorname{rank}(G / A)\right\}$. Since $A$ and $B$ are disjoint basic subgroup of $G$ we know $G$ cannot be bounded. Now $(G / A)[p] \supset[(A \oplus B) / A][p]$ and $|[(A \oplus B) / A][p]|=|B|$ which must be at least $\aleph_{0}$. Thus $\operatorname{rank}(G / A) \geqq \aleph_{0}$, and therefore

$$
|L|=\operatorname{rank}(G / A)<|G| \text {. }
$$

We can write each $x \in B$ as $x=y_{x}+f_{x}$, where $y_{x} \in L$ and $f_{x} \in F$. Since $|B|=|A|=|G|>|L|$ and $B$ is a subgroup, there must exist some $y \in B$ such that $y \in F$, but $F \subset A$ which contradicts $A \cap B=0$. Thus $\operatorname{rank}(G / A)=|G|$, and similarity rank $(G / B)=|G|$.

We are now in a position to state the results of the original questions in Theorem 6 and Theorem 7.

THEOREM 6. A necessary and sufficient condition for a reduced Abelian p-group to possess disjoint basic subgroups is that final $\operatorname{rank}(G)=|G|$.

Proof. If final $\operatorname{rank}(G)=|G|$ then Theorem 3 completes the proof. If $A$ and $B$ are disjoint basic subgroups of $G$ then by Theorem 5 we have $r(G / A)=r(G / B)=|G|$. But final $\operatorname{rank}(G) \geqq$ $\operatorname{rank}(G / M)$ for any basic subgroup $M$ of $G$. Thus final $\operatorname{rank}(G) \geqq$ $\operatorname{rank}(G / A)=|G|$, and since $|G| \geqq$ final rank $(G)$ we have final rank $(G)=|G|$.

THEOREM 7. If $G$ is a reduced Abelian p-group such that final $\operatorname{rank}(G)=|G|$, and $A$ is a basic subgroup of $G$, then there is a basic subgroup of $G$ which is disjoint from $A$ if and only if $A$ is a lower basic subgroup of $G$.

Proof. If $A$ is a lower basic subgroup then Lemma 2 assures the existence of a disjoint basic subgroup. If $G$ possesses a basic 
subgroup $B$ disjoint from $A$ then by Theorem 5 we have $\operatorname{rank}(G / A)=$ $|G|$ and by hypothesis final $\operatorname{rank}(G)=|G|$ thus $\operatorname{rank}(G / A)=$ final rank $(G)$ and $A$ is a lower basic subgroup.

\section{BIBLIOGRAPHY}

1. L. Fuchs, Abelian Groups, Pergamon Press, New York, 1960.

2. John M. Irwin, High subgroups of abelian torsion groups, Pacific J. Math. 11, (1961), 1375-1384.

3. Irving Kaplansky, Infinite Abelian Groups, The University of Michigan Press, Ann. Arbor, 1954.

4. A. Richard Mitchell, and Roger W. Mitchell Some structure theorems for infinite abelian p-groups. Journal of Algebra vol. 5, no. 3 (1967), 367-372.

Received July 14, 1966. 



\section{PACIFIC JOURNAL OF MATHEMATICS}

\section{EDITORS}

\section{H. ROYDEN}

Stanford University

Stanford, California

J. P. JANS

University of Washington

Seattle, Washington 98105

\section{J. DUGUNDJI}

Department of Mathematics

Rice University

Houston, Texas 77001

RICHARD ARENS

University of California

Los Angeles, California 90024

ASSOCIATE EDITORS

E. F. BECKENBACH

B. H. NeumanN

F. WoLF

K. YosidA

\section{SUPPORTING INSTITUTIONS}

\author{
UNIVERSITY OF BRITISH COLUMBIA \\ CALIFORNIA INSTITUTE OF TECHNOLOGY \\ UNIVERSITY OF CALIFORNIA \\ MONTANA STATE UNIVERSITY \\ UNIVERSITY OF NEVADA \\ NEW MEXICO STATE UNIVERSITY \\ OREGON STATE UNIVERSITY \\ UNIVERSITY OF OREGON \\ OSAKA UNIVERSITY \\ UNIVERSITY OF SOUTHERN CALIFORNIA
}

\author{
STANFORD UNIVERSITY \\ UNIVERSITY OF TOKYO \\ UNIVERSITY OF UTAH \\ WASHINGTON STATE UNIVERSITY \\ UNIVERSITY OF WASHINGTON \\ AMERICAN MATHEMATICAL SOCIETY \\ CHEVRON RESEARCH CORPORATION \\ TRW SYSTEMS \\ NAVAL ORDNANCE TEST STATION
}




\section{Pacific Journal of Mathematics}

\section{Vol. 23, No. 1 \\ March, 1967}

M. J. C. Baker, A spherical Helly-type theorem ................... 1

Robert Morgan Brooks, On locally m-convex*-algebras.............. 5

Lindsay Nathan Childs and Frank Rimi DeMeyer, On automorphisms of separable algebras ...................................

Charles L. Fefferman, A Radon-Nikodym theorem for finitely additive set

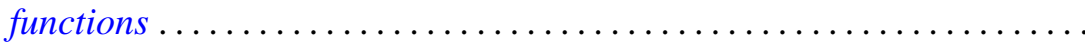

Magnus Giertz, On generalized elements with respect to linear

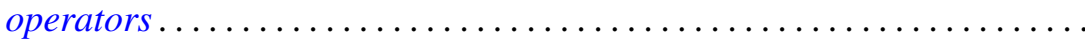

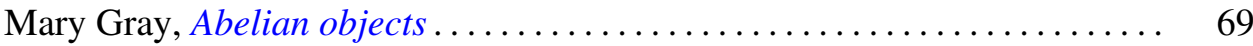

Mary Gray, Radical subcategories.............................. 79

John A. Hildebrant, On uniquely divisible semigroups on the two-cell . . . . . 91

Barry E. Johnson, AW*-algebras are $\mathrm{QW}^{*}$-algebras ............... 97

Carl W. Kohls, Decomposition spectra of rings of continuous functions . . . . 101

Calvin T. Long, Addition theorems for sets of integers .............. 107

Ralph David McWilliams, On $w^{*}$-sequential convergence and quasi-reflexivity ................................... 113

Alfred Richard Mitchell and Roger W. Mitchell, Disjoint basic

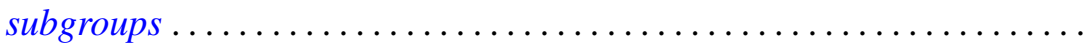

John Emanuel de Pillis, Linear transformations which preserve hermitian and positive semidefinite operators .

Qazi Ibadur Rahman and Q. G. Mohammad, Remarks on Schwarz's lemma

Neal Jules Rothman, An $L^{1}$ algebra for certain locally compact topological semigroups ...

F. Dennis Sentilles, Kernel representations of operators and their adjoints ...

D. R. Smart, Fixed points in a class of sets

K. Srinivasacharyulu, Topology of some Kähler manifolds

Francis C.Y. Tang, On uniqueness of generalized direct decompositions .

171 Albert Chapman Vosburg, On the relationship between Hausdorff dimension and metric dimension . 